\title{
ANÁLISIS BIOGRÁFICO DEL DIVORCIO EN ESPAÑA ${ }^{1}$
}

\section{René Houle, Carles Simó, Montserrat Solsona y Rocío Treviño Universitat Autónoma de Barcelona \\ E-mail: rhoule@cedserver.uab.es}

\begin{abstract}
RESUMEN
A partir de una explotación de los datos de la Encuesta Sociodemográfica de 1991, se presentan los resultados principales de una investigación en curso sobre los determinantes sociodemográficos del divorcio en España. Se tratará de explicar la propensión a la ruptura de una primera unión, configurada como variable dependiente, en función de un conjunto de covariables que harán referencia a características de la familia de origen, a características individuales y, por último, a las características de la unión. Se medirá el efecto de estas covariables sobre la probabilidad de la experiencia de una persona en la disolución de su primera unión, con el objetivo de realizar una primera aproximación al perfil sociodemográfico de la separación y el divorcio en España. El análisis realizado utiliza un modelo de azar de regresión logística discreto.
\end{abstract}

1 Este texto es un intento de sintetizar los resultados más relevantes obtenidos hasta ahora en una línea de investigación sobre el divorcio existente en el Centre d'Estudis Demogràfics desde enero de 1997 y que contó en sus inicios con un apoyo financiero del Institut Català de la Dona. Está precedido, por tanto, de otros textos en los que se describen de forma mucho más pormenorizada los resultados de cada una de las etapas del proceso de análisis realizado del divorcio:

- M. Solsona, C. Simó y R. Houle (1997): «Séparation et divorce en Espagne», XXIIIrd General Population Conference, IUSSP, Beijing, China (está publicado en Papers de Demografia, núm. 129, Centre d'Estudis Demogràfics).

- R. Houle, C. Simó, M. Solsona y R. Treviño (1997): Informe analitic de la població separada y divorciada a Catalunya, pp. 1-32 (informe realizado para el Institut Català de la Dona en razón de un convenio de colaboración, mimeo).

\section{Reis}




\section{INTRODUCCIÓN: ETAPAS, METODOLOGÍA Y PRINCIPALES HIPÓTESIS}

Se presentan aquí los principales resultados de una línea de investigación sobre el divorcio iniciada en el Centre d'Estudis Demogràfics en enero de 1997. Los trabajos realizados hasta ahora dibujan tres etapas en el análisis del divorcio, y cada una de ellas ha representado un paso adelante tanto en la metodología y sofisticación del análisis utilizado como en relación a las variables sociodemográficas contempladas para configurar una primera sociodemografía del divorcio en España.

En una primera fase se utilizó como fuente estadística principal el fichero de microdatos del Censo de Población de 1991, que contiene una muestra del mismo del 10 por 100 , con la pretensión de analizar el perfil sociodemográfico de la población divorciada y separada. El análisis se centró en la población divorciada y separada residente en Cataluña. En esta ocasión no se hizo referencia a la población del conjunto del Estado, pues se comprobó que el perfil no era muy distinto al de la población de Cataluña. En primer lugar se cuantificaron los efectivos y en segundo se compararon las características sociodemográficas de la población separada y divorciada en relación a la población casada, controlando la edad y el sexo. Las variables contempladas fueron el nivel de instrucción, la relación con la actividad, la profesión, la situación profesional y el tamaño de municipio. Se trataba de averiguar si la población separada y divorciada presentaba un perfil específico en relación a la casada que pudiera denotar un cierto proceso de selección social de la población susceptible a padecer un divorcio o separación.

En la segunda fase, como en la tercera, el análisis se basó principalmente en la explotación del fichero de microdatos de la Encuesta Sociodemográfica de 1991, que es una fuente privilegiada para el estudio de los eventos demográficos desde una perspectiva biográfica, ya que contiene información retrospectiva sobre la biografía familiar, educativa, laboral y migratoria de cerca de 156.000 individuos de 10 y más años de edad y, además, es representativa para cada Comunidad Autónoma. Se trabajó con un fichero seleccionando las primeras uniones del conjunto del Estado, ya fueran matrimoniales o de cohabitantes, que eran cerca de 108.000 y entre las cuales se registraba un total de 4.020 rupturas (ya fueran divorcios o separaciones legales o de hecho).

En esta segunda fase de análisis, y también en la tercera, el propósito era ya abordar los determinantes sociodemográficos del divorcio o, mejor dicho, de las rupturas de las primeras uniones en España, si bien en primer lugar se trató

- M. Solsona, H. René y C. Simó (1997): «Separation and Divorce in Spain», pp. 1-31 (de próxima publicación en la revista South European Society \& Politics).

Este texto constituye la ponencia escrita presentada en el VI Congreso Español de Sociología, en el grupo de trabajo 04, "Sociología de la Familia», y en la primera sesión de trabajo, «Pautas de Formación de la Familia» (A Coruña, 24-26 de septiembre de 1998). 
de medir la intensidad del fenómeno y sus patrones (las probabilidades de ruptura por promoción de la unión según duración de la misma por sexo y la edad media en el momento de la ruptura por promoción de la unión).

Los determinantes sociodemográficos del divorcio contemplados intentaban abarcar las dimensiones de estudio del fenómeno: el origen familiar, las características de las uniones y, por último, las características individuales. La mayoría de los cálculos se hicieron utilizando el análisis de las tablas de vida en un primer momento. La técnica de análisis de las tablas de vida consiste en dividir el período de observación (en el caso que nos ocupa, desde el principio de la unión hasta su disolución, por ruptura, por muerte del cónyuge o por alcanzar el momento de la Encuesta) en pequeños intervalos de tiempo (que en este caso eran anuales y se correspondían con la duración de la unión). Para cada intervalo se calcula la probabilidad de ocurrencia de un evento terminal (en nuestro caso, la ruptura). De esta forma, las probabilidades de ocurrencia del evento (de la ruptura) se estimaron para cada duración de la unión, lo cual permitió el cálculo de las tasas de riesgo de ruptura para cada duración de la unión. De este modo, fueron calculándose las proporciones de las primeras uniones supervivientes a una ruptura (controlando casi siempre la cohorte de la unión y su duración) según las diferentes categorías en que se subdividían las variables independientes tomadas como referencia y que intentaban abarcar el análisis de las tres dimensiones del fenómeno ya mencionadas.

Por último, en lo que podría ser clasificado como una tercera fase en la metodología de investigación, se emprendió el análisis de los determinantes sociodemográficos del divorcio manteniendo las tres dimensiones de referencia (el origen familiar, características de las uniones y características individuales) en base a modelos de riesgo de tiempo discreto utilizando la regresión logística. En relación a la metodología anterior suponía un avance analítico importante, pues ya no se estudiaba sólo el efecto de cada variable por separado sobre la ocurrencia del fenómeno, sino que permitía encuadrar en un mismo modelo de análisis el conjunto de covariables, de forma que las probabilidades de ocurrencia de la ruptura según una variable determinada están condicionadas por la acción conjunta del resto de las variables incluidas en el modelo. Por otro lado, las variables de referencia de algunas de las dimensiones se enriquecieron.

Esta metodología de análisis implicó la creación de un nuevo fichero en el que la variable dependiente se codificó como variable dicotómica (si el sujeto había padecido una ruptura o no desde el inicio de la unión) y los individuos fueron seguidos año a año desde el inicio de la unión hasta la ruptura, el fallecimiento del cónyuge o la fecha final de la encuesta. Para cada año en el que el individuo estaba a riesgo de padecer un divorcio, se creaba un registro de observación separado. De forma que si el fichero inicial contaba con cerca de 108.000 registros, correspondientes al número de uniones, el fichero final de trabajo se amplió a cerca de 2.600 .000 registros, correspondientes al número de años-persona en que los individuos estaban a riesgo de padecer una ruptura. 
Para cada año-persona, la variable dependiente (la transición de estar en unión a su ruptura, ya sea por divorcio o separación legal o de hecho) se codificaba con 0 si permanecía en unión en ese año particular y con 1 si en ese año se producía la ruptura. Aquellos individuos cuya información biográfica estaba censurada a la derecha (no protagonizaron nunca una ruptura) contribuían con el total de años en que eran observados hasta el momento de la encuesta, al igual que los individuos que no padecían una ruptura desde la unión hasta el momento de la muerte del cónyuge.

Finalmente, a las covariables o variables explicativas se les asignaron los valores que tomaban en cada año-persona analizado. Como en la Encuesta Sociodemográfica la datación de los acontecimientos no es muy precisa (sólo da información sobre el año de ocurrencia del fenómeno, no sobre el mes y el día), se nos presentaba el problema del orden secuencial de los acontecimientos que se hubiesen producido en un mismo año. Así, por ejemplo, este problema fue particularmente notable en los numerosos casos en los que el año del divorcio coincidía con el año de entrada en actividad de una mujer. No sabíamos con precisión si el divorcio provocaba la entrada en actividad o, al contrario, si la entrada en actividad precondicionaba el divorcio. Para esquivar este problema, en los casos en que el año de inicio de actividad coincidía con el año de ruptura de la unión, se codificó como 0. Por último, el conjunto de años-persona se trató como una sola muestra y se le estimó los modelos logísticos para nuestra variable dicotómica (experimentar una ruptura de la unión) usando el método de estimación de la máxima verosimilitud. Por tanto, nuestra variable dependiente se explicó por el conjunto de covariables incluidas en el modelo.

Cada una de estas etapas en análisis del divorcio partió de la formulación de una serie de hipótesis que conforme avanzaba la investigación se iban ampliando y se tornaban más específicas. Pero la principal hipótesis de partida era que, a diferencia de lo se señalaba en otros contextos donde el divorcio asume una intensidad mucho mayor y cuenta con una historia más larga, en España, al igual de lo que se conoce del caso italiano, donde la legislación sobre el divorcio es reciente y además las rupturas de las uniones registran un nivel mucho más bajo, el divorcio o, mejor dicho, los procesos de ruptura de las uniones se presentarían como un fenómeno social selectivo y menos normalizado como elemento estructural de la concepción de las relaciones de pareja.

En segundo lugar, si la literatura reciente enfatizaba como factores determinantes del divorcio los factores individuales (como, por ejemplo, una edad temprana al matrimonio), en el caso de España se formularon como hipótesis, además, que factores de índole contextual (como el efecto de la generación, de la introducción de la reciente legislación sobre el divorcio, como las características de la familia de origen, como la región de pertenencia, etc.) jugarían asimismo un rol cuanto menos igual o de mayor relevancia en la determinación de las rupturas.

Y en tercer lugar, dado que, por un lado, en España la política social tiene 
como telón de fondo la familia más que el individuo y que principalmente los derechos sociales se derivan de la participación en el mercado de trabajo; y que, por otro, no existen medidas específicas para paliar los efectos que a nivel individual puedan generarse cuando acontece un divorcio en situación de desigualdad de género en el ámbito laboral y familiar, la independencia económica de las mujeres se presentó, a nivel hipotético, como uno de los factores individuales clave en la determinación de la entrada en una ruptura de la unión de las mismas.

Finalmente, un último interrogante, que se planteó en la última etapa de análisis, era saber si los factores determinantes del divorcio podían haber cambiado en las cohortes de ruptura de la unión más recientes en relación a los de las cohortes más antiguas. En este sentido, podría esperarse que las variables de origen familiar condicionaran, por ejemplo, en mayor medida el acceso al divorcio en las promociones más antiguas que lo que lo harían en las más recientes, donde cabría esperar mayor fuerza de las variables individuales (nivel educativo o relación de actividad).

\section{PRINCIPALES RESULTADOS}

Se expondrán aquí los resultados más relevantes del trabajo ya realizado (ver nota 1), además de introducirse los resultados del análisis de regresión logística sobre los determinantes sociodemográficos de la propensión al divorcio.

\subsection{En relación a la intensidad de las rupturas de las uniones y su evolución en España}

En 1996, las estadísticas del Consejo General del Poder Judicial señalan que en España se iniciaron 51.317 procesos de separación y 32.571 procesos

2 La inexistencia de un registro de divorcios constituye uno de los principales obstáculos con los que se encuentra el investigador que quiere medir la intensidad del divorcio en España. La única fuente que proporciona una estimación directa del número de divorcios son las «Memorias Anuales del Consejo General del Poder Judicial», pero éstas se limitan a publicar el número de procesos de separación y divorcio (no de sentencias) iniciados cada año y si son litigiosos o no, en cada Comunidad Autónoma. De forma indirecta, se cotejó el número de procesos de divorcio y separación que las Memorias del Consejo señalaban que se habían iniciado entre 1981-1991 en España con el número estimado de éstos, tomando como referencia la población de divorciados de 1981, 1986 y 1991 de los censos y padrones de población y asimilando que los divorcios producidos durante el período eran igual a la población divorciada del último año, menos la población divorciada del año inicial, más los matrimonios de segundas nupcias de divorciados (obtenibles en las estadísticas del Movimiento Natural de la Población), más las defunciones de divorciados producidas durante el período (obtenibles también en las estadísticas del Movimiento Natural). El resultado mostró que el método de las estadísticas vitales subestimaba muchísimo el número de divorcios. Dada la pobreza de los datos proporcionados por las Memorias del Consejo (ni siquiera están disponibles los divorcios y separaciones por duración de matrimonio ni por 
de divorcio. Teniendo en cuenta los matrimonios que, según el Movimiento Natural de la Población Española, se produjeron ese mismo año en España, se contabilizaban 26 procesos de separación y 16 procesos de divorcio por 100 matrimonios celebrados en 1996.

Un mejor indicador comparativo de la intensidad del divorcio es el Índice Sintético de Divorcialidad, por cuanto permite comparar el número de matrimonios que acaban en divorcio en un momento dado en poblaciones con diferentes estructuras de divorcialidad por duración de la unión. En España, según los datos de la Encuesta Sociodemográfica de 1991, y tomando como referencia el período 1986-91, de cada 100 primeros matrimonios, 13 acaban en ruptura.

\section{TABLA 1}

\section{Indice Sintético de Divorcialidad. Paises europeos, 1990}

\begin{tabular}{|c|c|c|c|}
\hline Países & $\begin{array}{l}\text { Indice Sintético } \\
\text { de Divorcialidad } \\
\text { (por } 100 \\
\text { matrimonios) }\end{array}$ & Países & $\begin{array}{c}\text { Indice Sintético } \\
\text { de Divorcialidad } \\
\text { (por } 100 \\
\text { matrimonios) }\end{array}$ \\
\hline Estonia ........ & 46 & Moldavia .. & 30 \\
\hline Letonia ............. & 44 & Holanda .. & 28 \\
\hline Dinamarca ....................... & 44 & 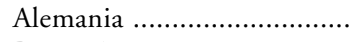 & 27 \\
\hline 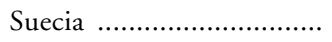 & 43 & 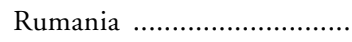 & 19 \\
\hline Inglaterra y Gales ............. & 42 & 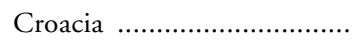 & 17 \\
\hline Finlandia .......................... & 41 & 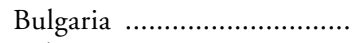 & 17 \\
\hline Noruega ............................... & 40 & 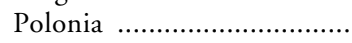 & 15 \\
\hline República Checa ............. & 38 & 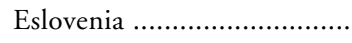 & 14 \\
\hline Luxemburgo ........................ & 36 & 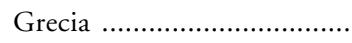 & 13 \\
\hline Lituania ............................. & 34 & España $(1986-90)$................ & $13^{*}$ \\
\hline 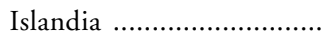 & 34 & Portugal (1989) ..................... & $12^{* *}$ \\
\hline Suiza ............................... & 33 & Italia & 8 \\
\hline 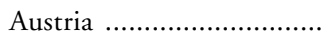 & 33 & 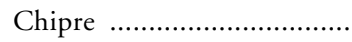 & 7 \\
\hline 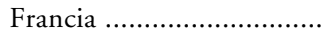 & 32 & 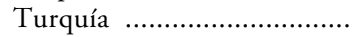 & 6 \\
\hline 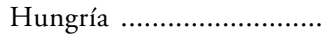 & 31 & 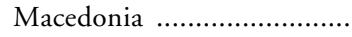 & 5 \\
\hline Bélgica ............................. & 31 & & \\
\hline
\end{tabular}

* Para España, el indicador se refiere a las disoluciones - divorcios y separaciones legales o de hecho- de primeros matrimonios (excluidas las cohabitaciones) del período 1986-90. Obviamente, se sobreestima el divorcio puesto que algunas separaciones no acabarían nunca en divorcio. Elaborado a partir de los datos de la Encuesta Sociodemográfica de 1991.

** Para Portugal, Monnier y Guibert-Lantoine (1993, p. 1063).

FuENTE: Consejo de Europa (1997, p. 41).

ninguna otra variable demográfica básica) y los pobres resultados de su estimación por el método de las estadísticas vitales, se emprendió el análisis del nivel, evolución y determinantes del divorcio mediante indicadores calculados principalmente a partir de los datos proporcionados por la Encuesta Sociodemográfica de 1991. 
En la tabla 1 puede verse que la incidencia del divorcio en España es muy baja si se compara con los niveles que se asumen en los países del norte de Europa, aunque es similar a la observada en otros países mediterráneos a finales de los ochenta.

Por otro lado, las diferencias de nivel por Comunidades Autónomas son acusadas. Así, el Índice Sintético de Ruptura de primeros matrimonios calculado para el período 1981-90 señala que el de Canarias es 4 veces mayor que el de Extremadura (20 por 100 versus 5 por 100). Los mayores niveles de ruptura de primeros matrimonios se registran en las comunidades isleñas y les siguen los de las comunidades más urbanizadas, Cataluña y Madrid. Los niveles de intensidad medios se localizan en Galicia, Asturias, País Vasco y Comunidad Valenciana. Por último, las comunidades del centro (excepto Madrid) y sur peninsulares muestran los más bajos niveles del indicador (ver tabla 2).

\section{TABLA 2}

Proporción de primeras uniones sobrevivientes a una ruptura, a diferentes duraciones de la unión, e Indice Sintético de Divorcialidad para el periodo 1981-90 por Comunidad Autónoma de residencia

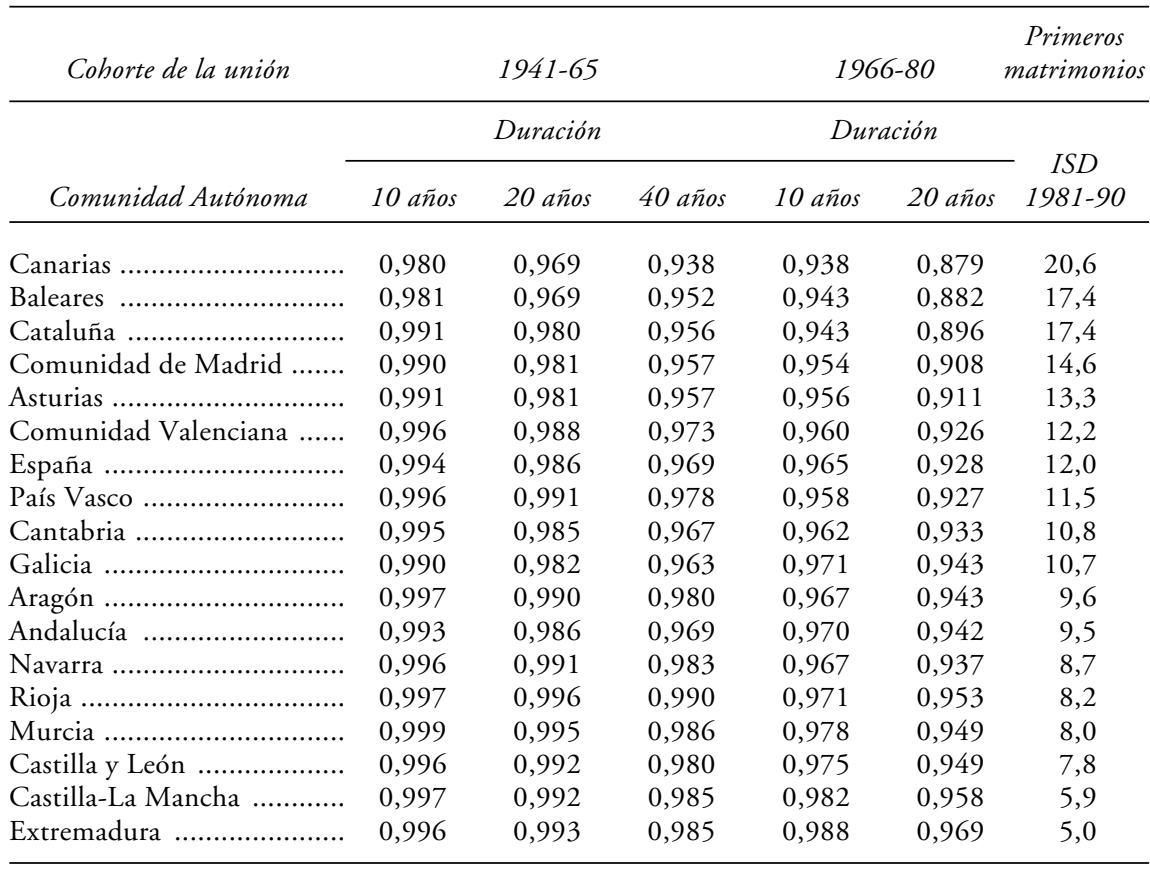

Fuente: Encuesta Sociodemográfica de 1991, INE. El Índice Sintético de Divorcialidad está calculado como en la tabla 1 (sólo para los primeros matrimonios, excluyendo las cohabitaciones) para el período 1981-90. 
La evolución de las rupturas de las uniones por cohorte de la unión, teniendo en cuenta la duración de la misma y el período histórico en el que se produce la ruptura, muestra que ya una década antes de la ley de aprobación del divorcio se inicia un cambio de tendencia de las rupturas al alza y que son unas determinadas generaciones las protagonistas de este cambio en el comportamiento nupcial. En el gráfico 1 pueden observarse las proporciones de supervivencia a una ruptura de la unión por cohorte de la unión y duración de la misma. En las cohortes de primeras uniones de 1971-75, constituidas principalmente por las generaciones de hombres y mujeres nacidos entre 1951 y 1955, un 5,8 por 100 ha acabado en ruptura. La incidencia de las rupturas de unión se dobla en estas cohortes en relación a la que experimentan las cohortes de 1956-60, entre las cuales sólo un 2,9 por 100 ha acabado en ruptura en el momento de la encuesta.

\section{GRÁFICO 1}

Proporción de primeras uniones sobrevivientes a una separación por duración de la unión. Promociones de unión 1941-45 a 1976-80. España

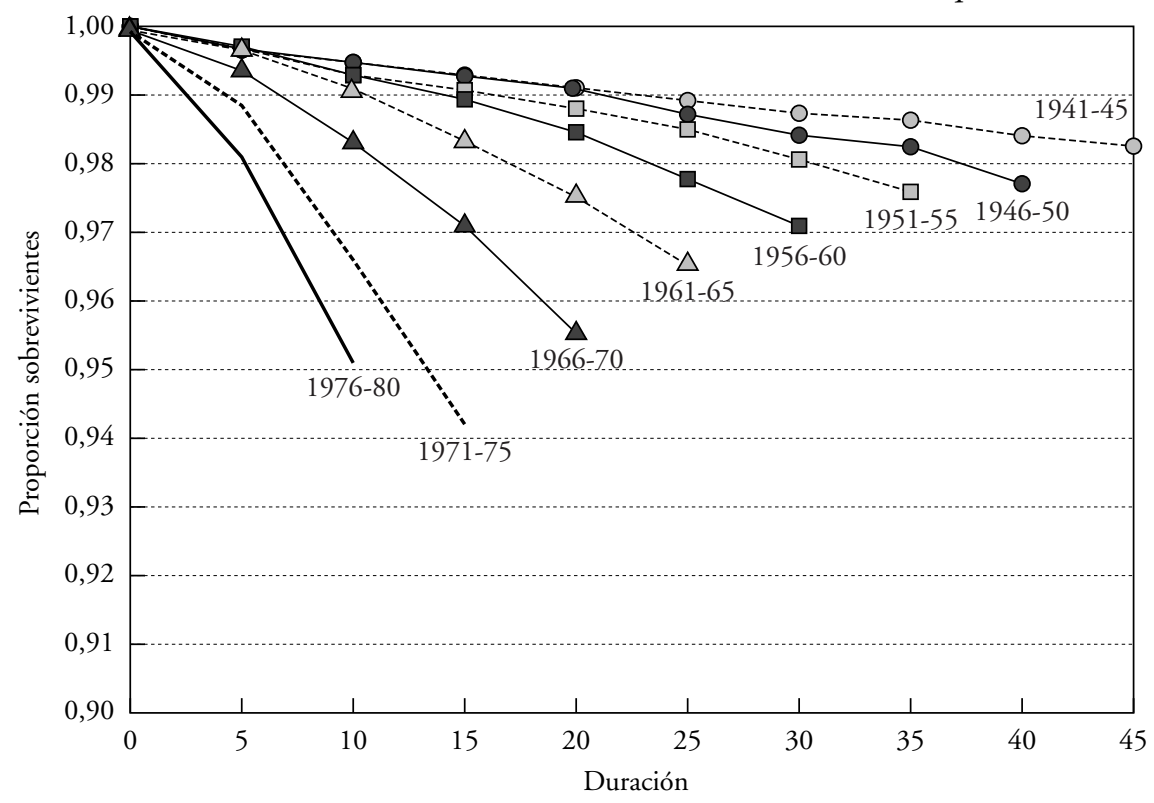

FUENTE: Encuesta Sociodemográfica de 1991, INE.

Las generaciones protagonistas de este cambio de tendencia al alza en el número de rupturas de las uniones, las generaciones nacidas entre 1946-50 y 1951-55, lo fueron también de los cambios políticos que envolvieron el final 
del régimen autoritario de Franco, al mismo tiempo que estuvieron en la vanguardia de nuevos comportamientos en el ámbito familiar que revelaban los primeros síntomas (el abrupto descenso de la fecundidad o la leve alza de la cohabitación) de que la Segunda Transición Demográfica empezaba su andadura en España. A partir de estas cohortes, las curvas de supervivencia de las uniones caen de forma constante a cualquier duración de la unión, lo que implica que la probabilidad de mantener intacta la unión es menor a medida que avanza el período histórico y duración de la unión.

Por último, en la tabla 3 y gráfico 2 puede observarse el doble efecto que tienen, por una lado, la cohorte de unión y, por otro, la duración en la tasas de riesgo. En la tabla 3, el efecto de la duración de la unión de cada promoción puede leerse en las columnas, y el efecto de las promociones o cohortes de las uniones para cada duración en las filas. Como puede observarse, las promociones de finales de los sesenta, protagonistas de los cambios socioculturales de la transición política, experimentan un incremento notable de las tasas de ruptura de la unión a cualquier duración de la misma.

\section{TABLA 3}

Tasas quinquenales de riesgo de ruptura de primeras uniones (por 10.000 uniones) por cohorte de unión y duración de la unión. España

\begin{tabular}{|c|c|c|c|c|c|c|c|c|c|}
\hline Duración (años) & $\begin{array}{c}1941- \\
1945\end{array}$ & $\begin{array}{c}1946- \\
1950\end{array}$ & $\begin{array}{c}1951- \\
1955\end{array}$ & $\begin{array}{l}1956- \\
1960\end{array}$ & $\begin{array}{c}1961- \\
1965\end{array}$ & $\begin{array}{l}1966- \\
1970\end{array}$ & $\begin{array}{l}1971- \\
1975\end{array}$ & $\begin{array}{r}1976- \\
1980\end{array}$ & $\begin{array}{c}1981- \\
1985\end{array}$ \\
\hline $0-4$ & 6,0 & 7,0 & 7,0 & 6,0 & 7,0 & 11,0 & 23,0 & 38,0 & 50,0 \\
\hline $5-9$ & 4,0 & 4,0 & 6,0 & 7,0 & 11,0 & 22,0 & 46,0 & 62,0 & \\
\hline 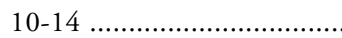 & 3,0 & 3,0 & 4,0 & 8,0 & 16,0 & 25,0 & 51,0 & & \\
\hline $15-19$ & 3,0 & 4,0 & 6,0 & 10,0 & 16,0 & 34,0 & & & \\
\hline $20-24$ & 5,0 & 7,0 & 6,0 & 14,0 & 20,0 & & & & \\
\hline 25-29 ….............................. & 4,0 & 6,0 & 9,0 & 14,0 & & & & & \\
\hline 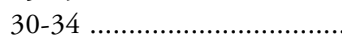 & 2,0 & 4,0 & 9,0 & & & & & & \\
\hline 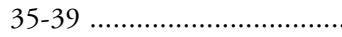 & 4,0 & 10,0 & & & & & & & \\
\hline 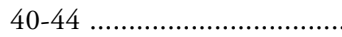 & 3,0 & & & & & & & & \\
\hline
\end{tabular}

FUENTE: Encuesta Sociodemográfica de 1991, INE.

Esta pauta de incremento será seguida por las sucesivas y más recientes promociones. El efecto de momento de la aplicación de la ley de divorcio de 1981 es visible en el gráfico 2, aunque, como puede observarse, es mucho menor de lo que cabría esperar. El cambio de comportamiento en relación a las rupturas de las uniones parece anticiparse a la futura ley. En efecto, en los períodos 1971-75 y 1976-80 se observa ya un incremento de las tasas de riesgo de ruptura de la unión, sobre todo en unas determinadas promociones. Parece, pues, que los efectos de cohorte son más decisivos que los efectos de momento 
ligados a la promulgación de la ley de divorcio (perceptibles por la acumulación de disoluciones que se produce en el momento de aprobación de la ley) a la hora de explicar la evolución reciente de las tasas de ruptura, si bien probablemente no puede descartarse una interacción de los dos factores.

Por último, cuanto más recientes son las generaciones o promociones de unión, más grande es la probabilidad de ruptura a cualquier duración de la unión. Sin embargo, entre las promociones más antiguas y más recientes parece dibujarse un cambio de patrón: si en las primeras el riesgo de ruptura parece cambiar poco de una duración de ruptura a otra, en las de más reciente formación los riesgos parecen ser mucho mayores a mayor duración de la unión, aunque a cualquier duración de la unión sean más grandes que en las promociones más antiguas.

\section{GRÁFICO 2}

Tasas quinquenales de riesgo de ruptura de primeras uniones (por 10.000 uniones) por duración de la unión en cada periodo correspondiente para las cohortes de uniones de 1941-45 a 1976-80. España

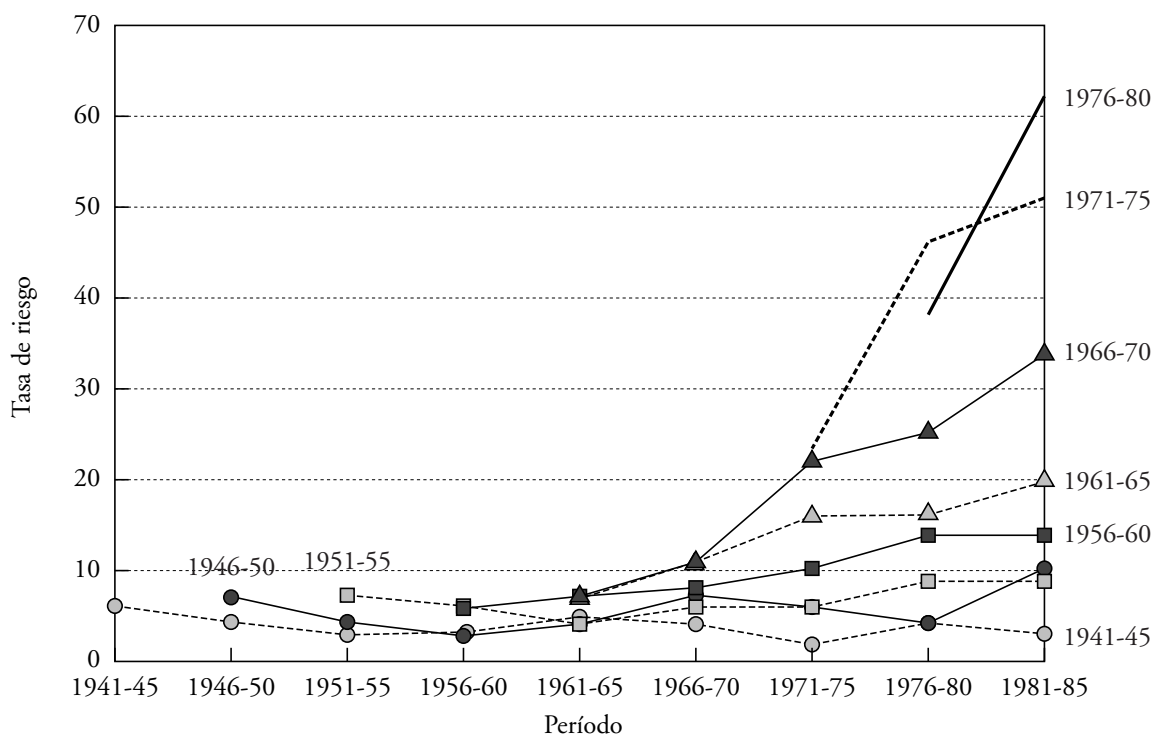

NotA: Cada línea representa una cohorte de unión que empieza con la duración 0-4 en el período correspondiente.

FUENTE: Encuesta Sociodemográfica de 1991, INE. 


\subsection{En relación al perfil sociodemográfico de la población divorciada y separada}

Como ya se ha mencionado, el perfil sociodemográfico de la población separada y divorciada se analizó en referencia a la población residente en Cataluña, puesto que en principio se empezó a trabajar en el tema de divorcio para el gobierno autónomo de Cataluña. Por otra parte, se realizó a partir de los datos del fichero de microdatos del Censo de 1991 y no del de la Encuesta Sociodemográfica del mismo año. De todas formas, se pudo comprobar que el perfil sociodemográfico no era muy diferente al que presentaba el conjunto de la población del Estado y, lo que es más importante, que el sentido de las diferencias entre la población separada, divorciada y casada era el mismo. Por esta razón, después del análisis de los efectivos de la población separada y divorciada del conjunto del Estado, se presenta a continuación el perfil sociodemográfico de la misma población residente en 1991 en Cataluña.

Según esta fuente, la importancia numérica, tanto en términos absolutos como relativos, de la población divorciada y separada (por situación legal y no de hecho) en esa fecha es pequeña: son 393.707 entre los 25 y 59 años y representan el 2,3 por 100 de la población de esas edades. De todas formas, el crecimiento relativo de estas subpoblaciones en los últimos quinquenios ha sido notable: la población separada se incrementa en un 236 por 100 entre 1975 y 1991, y es precisamente en el quinquenio que precede a la promulgación de la ley de divorcio cuando este incremento es de mayor magnitud. A partir de 1986 la población divorciada se incrementa a mayor velocidad que la separada.

Son relevantes los desequilibrios de stocks de la población divorciada por sexo, con una clara sobrerrepresentación de la femenina: por cada 100 divorciados de 25-29 años se registraban 167 divorciadas en 1991. Las diferencias en la frecuencia de segundas nupcias de uno y otro sexo es el principal factor explicativo de esta desigualdad de stocks. La tendencia de entrada a un nuevo matrimonio después de un divorcio es mucho más elevada entre los hombres que entre las mujeres: en relación al conjunto de matrimonios de población divorciada celebrados en España entre 1982 y 1994, hay 146 matrimonios de divorciados por 100 de divorciadas entre la población de 25 a 59 años, según los datos proporcionados por el Movimiento Natural de la Población Española publicado por el INE. Sin embargo, entre ambas fechas, los incrementos relativos de segundas nupcias de divorciadas son más intensos que los de divorciados, de forma que las diferencias de intensidad de segundas nupcias de divorciadas y divorciados disminuyen (en 1983 había 196 divorciados por 100 divorciadas, en 1986 este porcentaje era de 171, y en 1994 de 124 por 100).

Ahora bien, cuando las segundas nupcias no eran posibles debido a la no existencia de una ley de divorcio, las diferencias de stocks entre la población separada femenina y masculina también existían, lo que indica que factores como las diferencias de mortalidad por sexo y las existentes en la declaración 
de la situación separación y divorcio entre hombres y mujeres deben jugar un papel nada despreciable en la explicación de las diferencias de stocks ${ }^{3}$.

Cuando se considera el nivel de instrucción, la relación de actividad, la posición ocupada en el mercado de trabajo, tanto la categoría socioprofesional como la estabilidad en el trabajo, la población divorciada femenina residente en Cataluña presenta una situación ventajosa en relación a la población casada del mismo sexo. Ahora bien, cuanto más joven es la edad considerada, y por tanto más reciente es la generación de referencia, menores son estas diferencias entre la población divorciada y casada ${ }^{4}$.

Por otro lado, cuando se comparan en estos aspectos la población separada y divorciada femenina, parece que las divorciadas presentan un perfil socioprofesional concreto que las sitúa en mejor posición en la jerarquía social que a las separadas, en las que la similitud con las casadas es mayor (ver gráfico 3)

Efectivamente, en Cataluña, el porcentaje de mujeres sin estudios es entre las casadas de 25-59 años de edad de un 16 por 100, de un 14 por 100 entre las separadas, y de un 8 por 100 en las divorciadas. Por otro lado, el 16 por 100 de las casadas, el 21 por 100 de las separadas y el 36 por 100 de las divorciadas contaban en 1991 con un segundo o tercer grado de estudios. La vinculación al mercado de trabajo de las residentes en Cataluña de ese mismo grupo de edad también varía mucho según el estado civil: un 82,3 por 100 de las divorciadas se declara activo, frente a sólo un 45 por 100 de las casadas y un 78 por 100 de las divorciadas. Sin embargo, el paro golpea más a las separadas (22 por 100 de las activas) que a las casadas (19 por 100), y a las que menos a las divorciadas (18 por 100). La distribución de la población femenina ocupada por situación profesional señala también a la población divorciada como la más favorecida, seguida de la casada y, en tercer lugar, de la separada. Por un lado, el trabajo fijo está más expandido entre las ocupadas divorciadas (61 por 100) que entre las casadas (57 por 100), y todavía más que entre las separadas (55 por 100) de 25-59 años de edad, y, además, el peso de las «trabajadoras por cuenta propia con asalariados» también es ligeramente superior entre las divorciadas y casadas que entre las separadas; por otro lado, «las trabajadoras en cooperativas y trabajo familiar» y «las trabajadoras sin trabajo fijo», las dos categorías de situación profesional más bajas, concentran un mayor número de ocupadas entre la población separada, menor entre la casada y todavía más

3 En los cálculos que se hicieron en este trabajo con los datos de la Encuesta Sociodemográfica de 1991 se usó tanto la información referente a la población masculina como a la femenina, pero las declaraciones de ruptura de los hombres estaban claramente subestimadas en relación a las femeninas, y esto era especialmente relevante en las uniones recientes. Cuando se aplicó el método de las estadísticas vitales para estimar el número de separaciones y divorcios producidos entre dos años censales, también se registraba este fenómeno. La literatura especializada reciente ha subrayado este fenómeno de diferencias en la declaración del estado de divorciado por sexo (Castro et al., 1989).

${ }^{4}$ Aunque por razones de espacio no se presentan aquí estas diferencias por grupos de edad de más corta duración, pueden verse con este detalle en el informe presentado al Institut Català de la Dona referido en la nota 1. 


\section{GRÁFICO 3}

\section{Perfil sociodemográfico de la población femenina de 25-29 años por estado civil.} Cataluña, 1991

Nivel de instrucción

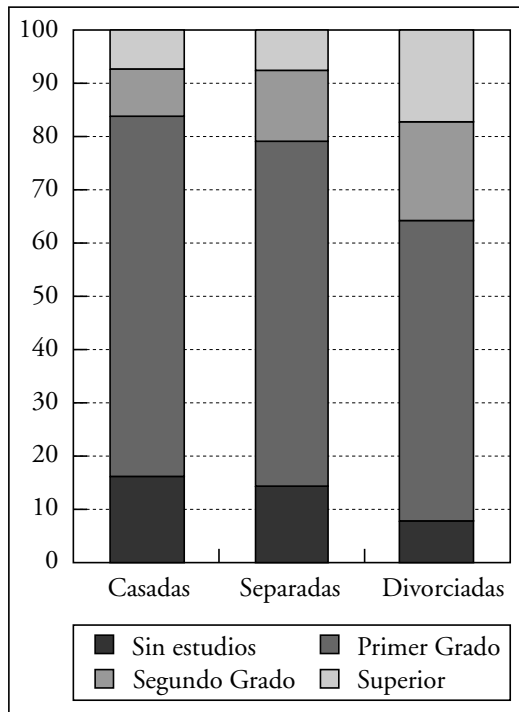

Grupo profesional

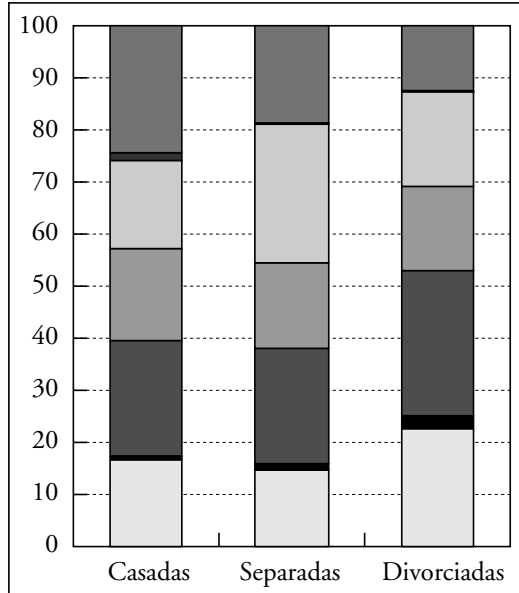

$\square$ Fuerzas Armadas $\square$ Trab. constr./indust.

$\square$ Trab. agrícolas $\square$ Servicios $\square$ Comerciales $\square$ Administrativas $\square$ Directivas $\square$ Técnicas
Relación de actividad

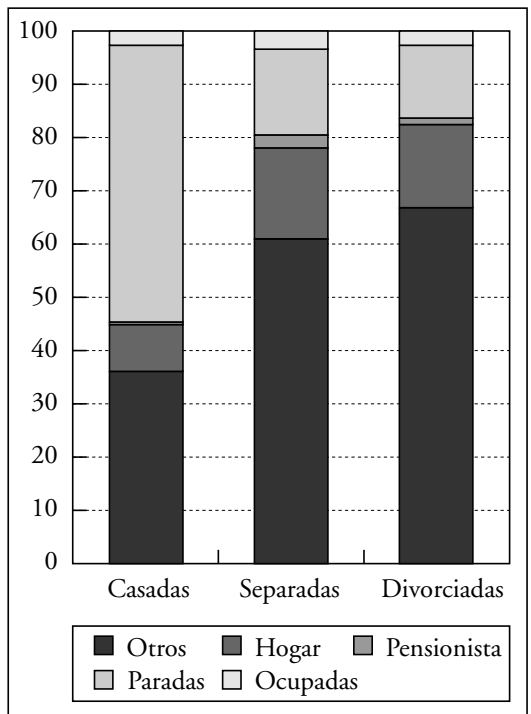

Situación profesional

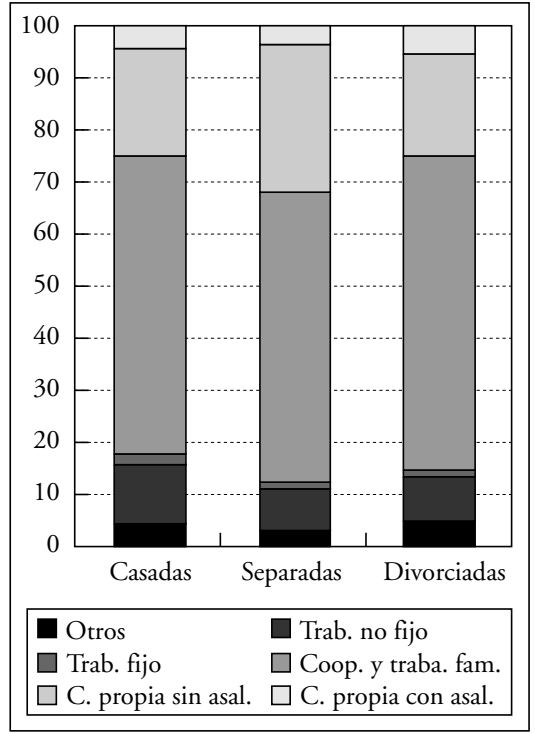

Fuente: Censo de Población de 1991. 


\section{GRÁFICO 4}

\section{Perfil sociodemográfico de la población masculina de 25-29 años por estado civil. Cataluña, 1991}

Nivel de instrucción

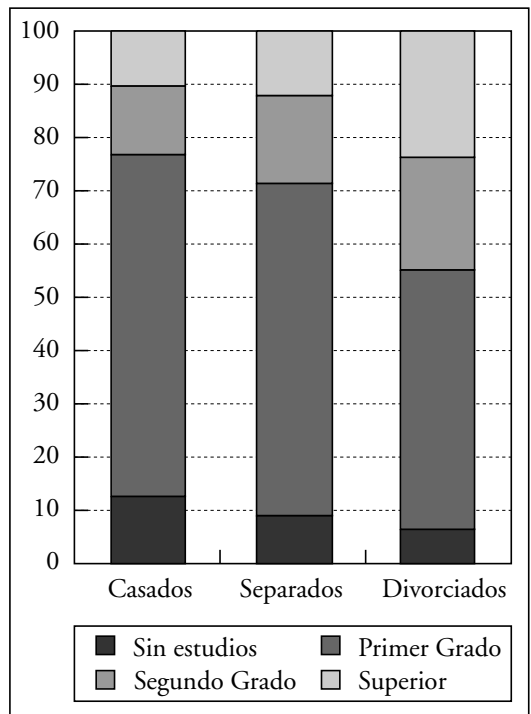

Grupo profesional

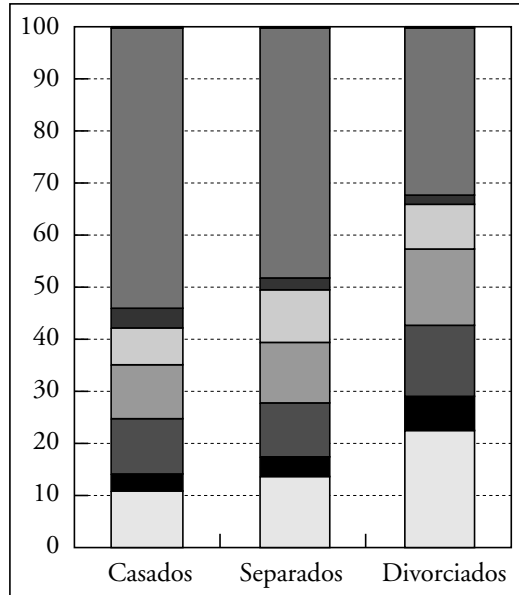

$\square$ Fuerzas Armadas $\mathbf{\square}$ Trab. constr./indust.

$\square$ Trab. agrícolas $\square$ Servicios $\square$ Comerciales

$\square$ Administrativos $\square$ Directivos $\square$ Técnicos
Relación de actividad

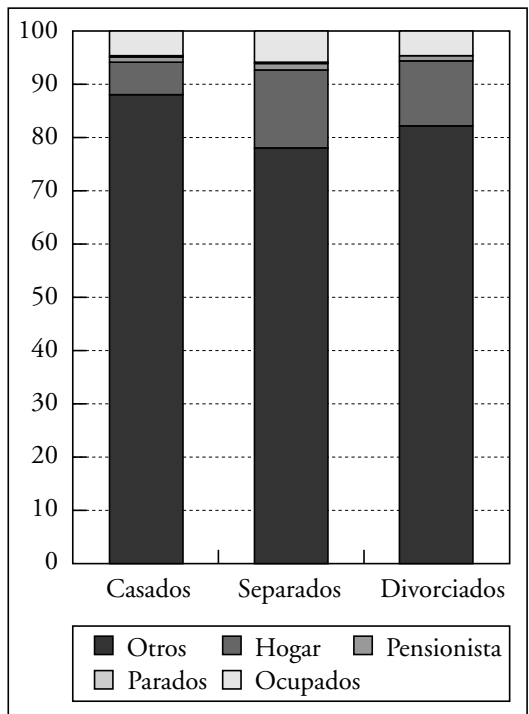

Situación profesional

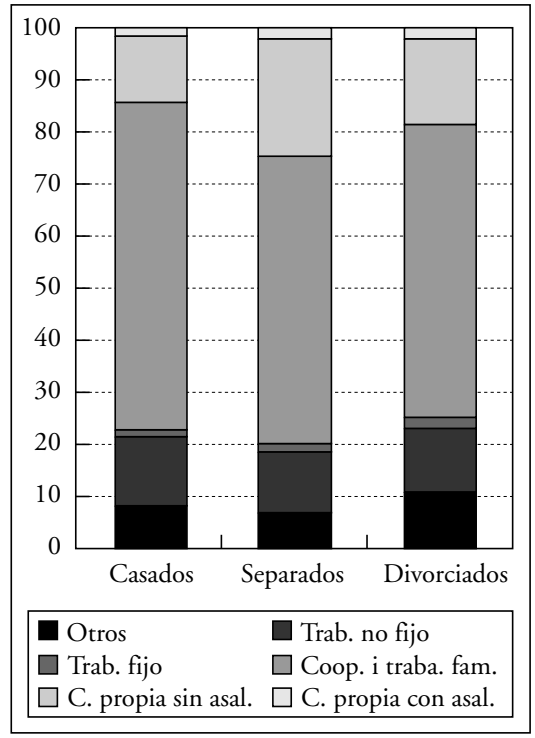

Fuente: Censo de Población de 1991. 
pequeña entre las divorciadas (un 30, 23 y 22 por 100 , respectivamente). Finalmente, también la población ocupada separada presenta más diferencias en la estructura por grupos de profesiones con la de las ocupadas divorciadas que con la de las casadas.

Si bien entre las separadas y divorciadas las profesiones del terciario tienen mayor peso que entre las casadas, entre las segundas, las técnicas, directivas y administrativas tienen mayor significado.

En 1991, los hombres en Cataluña también mostraban diferencias acusadas, incluso de mayor magnitud, en el nivel de instrucción según el estado civil. Un 23 por 100 de los divorciados de 25 a 59 años tenía un nivel de tercer grado, frente a sólo un 10 por 100 de casados y un 12 por 100 de separados, y, por otro lado, la categoría «sin estudios» concentraba sólo al 7 por 100 de los divorciados y, en cambio, a un 13 por 100 de casados. Sin embargo, en relación a la vinculación al mercado de trabajo, las diferencias por estado civil entre los hombres tienen un sentido muy diferente que entre las mujeres. Por un lado, la tasa de actividad de los divorciados y separados varía poco en relación a la de los casados y, además, contrariamente a lo que ocurría entre las mujeres, los hombres casados presentan un mayor nivel de ocupación, o sea, menos paro, que los divorciados y, sobre todo, más alto que el de los separados. La situación profesional y la estructura ocupacional entre los divorciados y separados es asimismo muy desigual, como ocurría entre las mujeres: si un 30 por 100 de los ocupados divorciados son técnicos o directivos, sólo lo es el 14 por 100 de casados; y en el otro extremo, las ocupaciones de la industria, construcción y trabajos agrícolas suponen en el primer caso el 32 por 100 de la ocupación y en el segundo, en cambio, el 54 por 100 (ver gráfico 4).

\subsection{En relación a los determinantes sociodemográficos de las rupturas}

El análisis de los determinantes sociodemográficos de las rupturas en España se basó principalmente en la explotación biográfica de la historia de las uniones de la Encuesta Sociodemográfica de 1991 y abarcó la segunda y tercera fases de análisis.

Como ya se ha mencionado, estos determinantes se agruparon en tres dimensiones: origen familiar, características de la unión y características individuales. Previamente a la realización de una regresión logística que estimase un modelo analítico que incluyera el tratamiento conjunto de las variables seleccionadas indicativas de cada dimensión se había estudiado, por otras metodologías, el efecto singular de la mayoría de variables en la propensión a una ruptura.

En relación al origen familiar, se calculó la proporción de primeras uniones sobrevivientes a una ruptura según se contara o no con el precedente de la ruptura de la unión de los padres en dos promociones de uniones (1941-65 y 1966-80) y a diferentes duraciones de la unión y, de este modo, se comprobó el efecto positivo que la ruptura de la unión de los padres tenía en la del sujeto. 
En referencia a las características de la unión, ya se ha comentado en el apartado de evolución la relevancia de la promoción de la unión en la determinación del nivel de ruptura. Otra de las cuestiones que mereció un estudio pormenorizado fue el efecto que podía tener la edad al matrimonio en las rupturas. Se calculó la edad media a la primonupcialidad por cohorte de matrimonio y también por generación de nacimiento y sexo, distinguiendo dos grupos: los matrimonios que habían sufrido una ruptura y aquellos otros que no la habían sufrido hasta 1991, el momento de la encuesta. Los resultados confirmaron las pautas que se habían observado en otros países europeos y en los Estados Unidos: en todas las promociones matrimoniales observadas, la proporción de matrimonios tempranos entre aquellos que habían experimentado una ruptura era mucho mayor que entre el grupo de matrimonios de la misma promoción y a la misma duración que no habían sufrido tal ruptura. El análisis de supervivencia por medio del método de las tablas de vida confirmó también estos resultados para las promociones de uniones de 1941-65 y 1966-80 en los dos sexos.

Otra de las características de la unión determinante de la entrada en una ruptura de la unión señalada en estudios especializados del divorcio, la diferencia de edad entre los cónyuges, también mereció un estudio particular. Se pudo comprobar que entre las uniones que habían sufrido una ruptura, las uniones que más se alejaban de la norma, en relación a la patriarcal y tradicional diferencia de edad entre los cónyuges, estaban más sobrerrepresentadas que entre el grupo de uniones que no habían experimentado ruptura. El cálculo de las proporciones de uniones sobrevivientes a una ruptura en diferentes promociones de la unión, a diferentes duraciones y para los sexos confirmaron estos resultados.

Si bien el tipo de unión (matrimonial o consensual) es otra de las variables comunes que aparece, en los estudios recientes de divorcio, como discriminatoria de diferentes probabilidades de que una unión acabe en ruptura, no se incluyó en el modelo de la regresión logística, aunque sí se analizaron sus efectos por medio de las tablas de vida (ver tabla 4).

Como puede comprobarse, la mayor inestabilidad de las uniones consensuales en relación a las matrimoniales es patente en ambas promociones y en todas las duraciones. Además, las diferencias parecen incrementarse no sólo con la duración de la unión, sino conforme es más reciente la cohorte de la unión.

En relación a las caracteristicas individuales, se comprobó que la duración media de la instrucción completa hasta el momento del matrimonio era más elevada en aquellos hombres y mujeres que habían sufrido una separación en todas las promociones de la unión. Esta misma variable también se analizó por el método de las tablas de vida, examinando las curvas de supervivencia a una ruptura en dos promociones, a diferentes duraciones de la unión y según los diferentes niveles de instrucción. Las conclusiones a que se llegaron confirmaron el carácter selectivo de las rupturas en España. 


\section{TABLA 4}

Proporciones de uniones supervivientes a una ruptura a diferentes duraciones de la unión por sexo del informante y cohorte de la unión. España

\begin{tabular}{|c|c|c|c|c|}
\hline Sexo y cohorte de la unión & $\begin{array}{l}\text { Duración } \\
\text { de la unión } \\
\text { (años) }\end{array}$ & Todos & Matrimonios & Cohabitaciones \\
\hline \multicolumn{5}{|l|}{ Hombres } \\
\hline \multirow[t]{3}{*}{$1941-65$} & 10 & 0,995 & 0,995 & 0,954 \\
\hline & 20 & 0,989 & 0,989 & 0,906 \\
\hline & 40 & 0,972 & 0,972 & 0,815 \\
\hline \multirow[t]{2}{*}{$1966-80$} & 10 & 0,969 & 0,971 & 0,764 \\
\hline & 20 & 0,940 & 0,942 & 0,715 \\
\hline \multicolumn{5}{|l|}{ Mujeres } \\
\hline \multirow[t]{3}{*}{$1941-65 \ldots}$. & 10 & 0,992 & 0,993 & 0,891 \\
\hline & 20 & 0,984 & 0,984 & 0,789 \\
\hline & 40 & 0,966 & 0,967 & 0,680 \\
\hline \multirow[t]{2}{*}{$1966-80$} & 10 & 0,961 & 0,963 & 0,738 \\
\hline & 20 & 0,917 & 0,920 & 0,617 \\
\hline
\end{tabular}

FUENTE: Encuesta Sociodemográfica de 1991, INE.

Por último, en esta fase exploratoria de variable a variable se puso especial énfasis en el análisis de la relación entre actividad económica femenina y ruptura de la unión. La razón de ello es que se había planteado como hipótesis que la independencia económica de las mujeres era uno de los factores individuales clave en la determinación de la ruptura de la unión. En un primer momento se trató de analizar la compleja relación entre ambas variables; si la población divorciada o separada femenina presentaba una mayor vinculación al mercado de trabajo podía ser que fuese: 1 ) porque la ruptura de una unión alentase la entrada en actividad; 2) o porque hubiese una preselección de la población susceptible de entrar en una ruptura, que precisamente seleccionase a la población que estuviera activa; 3) o, quizás, por ambas razones a la vez.

En el cuadro 5 pueden observarse los itinerarios de actividad que se diseñaron en función de la ruptura con el objetivo de analizar la relación entre actividad económica y ruptura de la unión. Entre las mujeres, los menos frecuentes son «el no haber estado nunca activa» $\mathrm{y}$ "el estar activa antes de la ruptura pero no después».

En cambio, el más común, y con diferencia, es el de aquellas mujeres que estaban activas antes, en el momento de la ruptura y después (un 56 por 100 de las mujeres unidas y separadas después de 1975 había seguido este itinerario). Así, pues, puede concluirse que si bien hay una clara preselección en favor de las activas de cara a la entrada en una ruptura de unión, también ésta alien- 


\section{TABLA 5}

\section{Itinerario de actividad en relación a la ruptura de la unión (en porcentajes). Uniones rotas después de 1974. España}

Mujeres Hombres

Itinerario laboral en relación a la ruptura de la unión.

Total de uniones rotas después de 1974

Activo/a antes, en el momento de la ruptura y después

56,0

85,8

Ha estado activo/a antes y después de la ruptura pero no en el año del evento

$11,1 \quad 2,6$

Activo/a el mismo año de la ruptura y después

10,1

1,0

Ha estado activo/a antes de la ruptura, después no

14,0

10,4

No ha estado nunca activo/a

8,8

0,2

TOTAL DE SEPARACIONES (1975 y después)

$100 \quad 100$

(1.744)

(1.211)

Intensidad laboral de la población todavía casada o viuda en 1991

Ha estado activo/a

71,2

99,9

No ha estado activo/a

28,8

0,1

TOTAL

FUENTE: Encuesta Sociodemográfica de 1991, INE.

ta algo la entrada en actividad: son minoritarios los itinerarios en que las mujeres habían estado activas antes de la ruptura pero no después; y, por otro lado, un 14 por 100 de mujeres (el segundo itinerario en frecuencia) estaban activas en el momento de la ruptura o después pero no antes, y otro 11 por 100 habían estado en algún momento de sus vidas activas antes de la ruptura, y si bien no lo eran en el año mismo, sí lo estaban después.

El estudio de la relación entre actividad económica y ruptura de la unión se enriqueció, a su vez, introduciendo el análisis de las tablas de vida y analizando la proporción de uniones sobrevivientes a una ruptura en la cohorte de uniones de 1966-80, según diferentes duraciones de la unión y para ambos sexos y distinguiendo tres grupos: aquellos individuos que en el momento de la unión trabajaban en el sector público, aquellos que lo hacían en el privado y los que eran ayudas familiares. Era interesante contemplar esta distinción ya que se podía suponer que, en términos de seguridad de empleo y derechos de seguridad social, los primeros tenían ventaja sobre los terceros, situándose en posición intermedia los segundos. En el caso de las mujeres la lectura era clara; existía un efecto positivo entre seguridad en el empleo y disolución de la unión: para esta promoción de la unión, 1966-80, y a los veinte años de duración, un 6 por 100 de uniones había sufrido una ruptura en el grupo de «ayu- 
das familiares», un 9 por 100 en el grupo de mujeres del sector privado y un 15 por 100 entre las del sector público.

Una vez realizada esta fase exploratoria variable a variable, se analizaron los determinantes sociodemográficos de las rupturas por medio de un modelo de regresión logística que incluía un tratamiento conjunto de las variables que se habían seleccionado como indicativas de las tres dimensiones: origen familiar, características de la unión y características individuales. Algunas de estas variables eran fijas: la categoría socioeconómica del padre del sujeto, la ruptura de la unión de los padres, la promoción de la unión, la edad en el momento de la unión y las diferencias de edad entre los cónyuges. En estos casos, el valor de la variable era el mismo a lo largo de los años transcurridos desde el momento de la unión hasta el fallecimiento del cónyuge o el momento de la Encuesta. Pero también se incluyeron variables cambiantes en el tiempo: el número de hijos, nivel de instrucción, la ocupación, tamaño de municipio o Comunidad Autónoma de residencia. En estas variables, en cada año-persona transcurrido, contabilizado a partir del momento de la unión hasta la ruptura, fallecimiento del cónyuge o momento de la encuesta, podía registrarse un valor diferente de la variable.

En la tabla 6 pueden observarse los resultados de la regresión logística en el colectivo de mujeres. Por un lado, disponemos de la frecuencia que asume cada valor de la variable contabilizado en años-persona. Por otro lado, se estima la probabilidad de ocurrencia del fenómeno para cada categoría de cada variable en relación a una categoría de referencia, que en todos los casos es la primera categoría de cada variable. Por último, se estima la significación estadística.

Cabe decir que la probabilidad de ocurrencia del evento está condicionada por cada una de las variables contenidas en el modelo. La categoría socieconómica del padre del sujeto, estimada cuando éste tenía 16 años, señala una relación positiva entre esta variable y la ruptura: a mayor categoría, mayor probabilidad de ruptura. Aquellas mujeres con un padre de categoría «media-alta» tienen un riesgo 15 por 100 menor de sufrir una ruptura de la unión que las que cuentan con un padre de categoría alta. El menor riesgo de ruptura se sitúa entre las que tienen un padre de categoría baja (35 por 100 menor que las de padre de categoría alta).

El precedente paterno de ruptura también favorece el divorcio. Aquellas mujeres que contaban con un padre o madre separado o divorciado tienen el doble de riesgo de experimentar una ruptura de la unión.

Como puede observarse en la misma tabla, la promoción de la unión es una variable que discrimina mucho el riesgo de padecer una ruptura. La última promoción tiene 5 veces más riesgo que la primera. Otra variable importante es la edad a la unión: el menor riesgo de ruptura se da entre aquellas que se casan a una edad elevada (un 60 por 100 menos de riesgo que las que lo hacen a una edad muy temprana), y el mayor entre las que se casan muy jóvenes.

La homogamia o heterogamia en relación a las diferencias de edad entre los cónyuges también se revela diferenciadora. El riesgo de ruptura no es muy distinto cuando la mujer es de menor edad que el varón en la unión (ya sea «6 o 


\section{TABLA 6}

Determinantes sociodemográficos de las rupturas. España.

Resultados de la regresión logistica sobre la ruptura de las primeras uniones.

$$
\text { Mujeres }
$$

\begin{tabular}{|c|c|c|c|}
\hline Variables sociodemográficas & $\begin{array}{l}\text { Años- } \\
\text { persona }\end{array}$ & $\operatorname{Exp}(B)$ & $\begin{array}{c}\text { Nivel } \\
\text { significación }\end{array}$ \\
\hline
\end{tabular}

Categoría socieconómica del padre

Alta

Media-alta

76.451

Media-baja

Baja

Sin clasificar

Ruptura de la unión de los padres

No

Sí

Promoción de la unión

1941-60

1961-75

1976-90

\section{Edad a la unión}

Temprana (<=21 años)

Media (22-26 años)

276.593

572.344

Elavada (27 y más)

Diferencia de edad entre los cónyuges

Misma edad o mayor el hombre

La mujer entre 5 y 1 año menor

La mujer 6 años o más menor

723.598

286.809

151.040

312.510

264.520

624.677

272.250

263.127

Número de hijos

Sin hijos

1 hijo

2 hijos

3 hijos

4 o más

Nivel de instrucción

Sin estudios

Primer grado

Segundo grado

Tercer grado

316.475

326.946

150.611

104.288

498.933

570.487

51.434

40.593

1

0,4757

0,3908

3,0986

5,0829

1

1,0876

1,5843

1

1,1935

0,8724

0,9373

1,1297

1

1,0817

1,4522

1,5673

809.371

352.076

$\underset{* *}{\text { Categ. Ref. }}$
$* * *$
$* * *$
NS

Categ. Ref.

$* * *$

Categ. Ref.

$* * *$

***

Categ. Ref.

Categ. Ref.

NS

***

Categ. Ref.

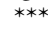

NS

NS

Categ. Ref.

NS

***

***

\section{Ocupación}

No

Sí
1

2,204
Categ. Ref. 


\section{TABLA 6 (continuación)}

Determinantes sociodemográficos de las rupturas. España.

Resultados de la regresión logistica sobre la ruptura de las primeras uniones.

$$
\text { Mujeres }
$$

\begin{tabular}{|c|c|c|c|c|}
\hline Variables sociodemográficas & $\begin{array}{l}\text { Años- } \\
\text { persona }\end{array}$ & $\operatorname{Exp}(B)$ & $\begin{array}{c}\text { Nivel } \\
\text { significación }\end{array}$ & \\
\hline \multicolumn{5}{|l|}{ Tamaño de municipio } \\
\hline 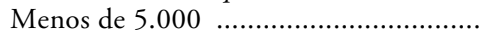 & 377.749 & 1 & Categ. Ref. & \\
\hline 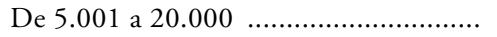 & 254.603 & 1,4042 & $* * *$ & \\
\hline 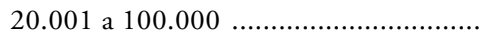 & 201.791 & 1,5779 & $* * *$ & \\
\hline 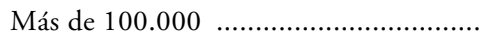 & 317.636 & 2,4918 & $* * *$ & \\
\hline 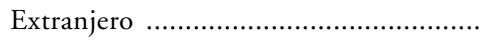 & 9.668 & 4,1976 & $* * *$ & \\
\hline \multicolumn{5}{|l|}{ Comunidad Autónoma } \\
\hline 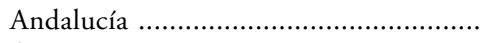 & 168.273 & 1 & Categ. Ref. & \\
\hline 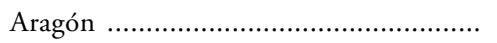 & 44.772 & 0,9303 & NS & \\
\hline 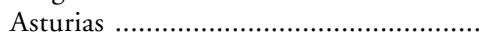 & 47.181 & 1,3234 & ** & \\
\hline 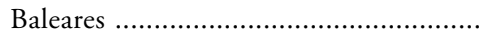 & 33.189 & 1,3363 & ** & \\
\hline 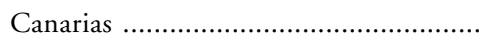 & 42.107 & 1,9405 & $* * *$ & \\
\hline 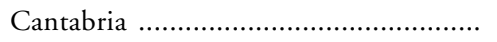 & 27.886 & 1,1822 & NS & \\
\hline Castilla-La Mancha ................................ & 83.755 & 0,8516 & NS & \\
\hline Castilla y León .................................... & 62.657 & 0,8132 & NS & \\
\hline 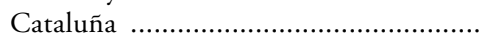 & 154.137 & 1,3877 & $* * *$ & \\
\hline Comunidad Valenciana ......................... & 99.918 & 1,1628 & NS & \\
\hline 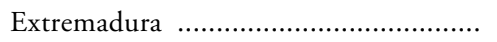 & 47.178 & 0,6023 & $* *$ & \\
\hline 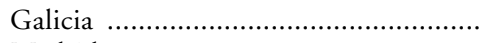 & 82.300 & 1,0345 & NS & \\
\hline 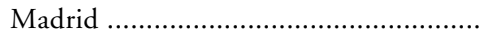 & 108.434 & 1,3665 & $* * *$ & \\
\hline Murcia & 38.831 & 0,5569 & $* * *$ & \\
\hline 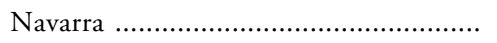 & 25.622 & 0,9772 & NS & \\
\hline 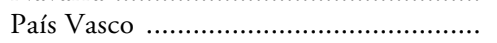 & 61.573 & 1,1068 & NS & \\
\hline 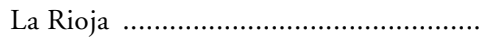 & 16.594 & 0,8065 & NS & \\
\hline Ceuta y Melilla ..................................... & 4.326 & 2,227 & $* * *$ & \\
\hline 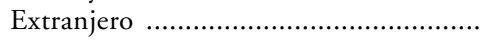 & 12.714 & 1,3399 & NS & \\
\hline \multirow{2}{*}{$\begin{array}{l}-2 \text { Log Likelihood } \\
\text { Goodness of Fit }\end{array}$} & 208 & Chi-Square & & 3277,455 \\
\hline & 5,02 & significación & & $\begin{array}{r}44 \\
* * *\end{array}$ \\
\hline
\end{tabular}

* Indica un nivel de significación de 0,01.

** Indica un nivel de significación de 0,05.

*** Uno de 0,1.

NotA: El modelo está controlado por la duración de la unión. 
más años menor» o "entre 5 y un año menor») y sí es significativamente mayor cuando es de igual edad o mayor que el marido.

La variable número de hijos es una variable que ha proporcionado muchos quebraderos de cabeza en esta investigación. La bibliografía reciente señala que en Estados Unidos las uniones sin hijos se asocian a mayores grados de inestabilidad, que el primer hijo reduce la probabilidad de divorcio, y que este efecto preventivo no se observa para los hijos de otro rango (Journal of Marriage and the Family, núm. 52, 19990, pp. 904-912). En Suecia, Anderson (1995) y Hoem (1997) señalan que tanto el número de hijos como la edad del más pequeño tienen un efecto en la propensión al divorcio, de forma que las parejas con dos o más hijos tienen un menor riesgo de experimentar un divorcio comparado con las que no tienen un hijo o sólo uno, y que cuanto más joven es el hijo menor, menor es la probabilidad de divorcio. En nuestro caso se han testado alrededor de una decena de diseños de esta variable en diferentes modelos de regresión logística, teniendo en cuenta el número de hijos y si el menor se situaba bajo el umbral de una u otra edad determinada. Los resultados han sido desiguales y poco clarificadores de una determinada tendencia. Puesto que se es todavía reacio a descartar un efecto claro del número de hijos y de la edad de éstos en la propensión al divorcio en España, se ha optado por incluir en el modelo que se presenta (ver tabla 6) la variable de la forma más sencilla, distinguiendo tan sólo el número de hijos, mientras no se resuelva y estudie esta cuestión con más profundidad.

En España parece dibujarse un patrón distinto: el riesgo de ruptura es mayor si se tiene un solo hijo que si no se tienen hijos, aunque este último es mayor que si se tienen dos hijos. El riesgo parece volver a incrementarse a partir de tres hijos o cuatro y más hijos, situándose al nivel del riesgo que tienen las que no tienen hijos.

En relación a las características individuales, el nivel de instrucción muestra en España un efecto positivo en la inestabilidad de las uniones. Las mujeres de tercer grado tienen un porcentaje de riesgo 157 por 100 mayor que las sin estudios o primer grado. Y la ocupación ${ }^{5}$ también muestra un efecto positivo en la propensión al divorcio.

Por último, se contemplan dos variables territoriales, que en esta ocasión no son fijas sino que varían a lo largo de la biografía de las mujeres, desde el momento de la unión hasta el de la ruptura, fallecimiento del cónyuge o momento de la encuesta, 1991. Si bien están contempladas en longitudinal, se codifican en cada año de la biografía de las mujeres tal y como el municipio se clasifica en tamaño en 1991 y tal y como se conforma actualmente la pertenencia de las provincias a las Comunidades Autónomas. Como puede obser-

5 En este caso, y para evitar el efecto acontecimiento de corto plazo, el examen biográfico anual de la relación de actividad ha comenzado también en el momento de la unión pero ha finalizado un año antes de la ruptura y no en el año de la misma, como se ha hecho con el resto de las variables aquí presentadas. 
varse, la residencia en municipios de más de 100.000 habitantes duplica el riesgo de ruptura en relación a la residencia en los municipios de menor tamaño, menores de 5.000 habitantes. A mayor tamaño de municipio, mayor riesgo de divorcio, si bien la residencia en el extranjero multiplica por cuatro el riesgo de ruptura en relación a la categoría de referencia, los municipios menores de 5.000 habitantes.

En el cuadro 6 puede observarse asimismo la importancia de la Comunidad Autónoma en la determinación de riesgos diferenciales de ruptura. La categoría de referencia es Andalucía, y en función de ella se estiman los riesgos proporcionales del resto de Comunidades. En Canarias casi se duplica, en Cataluña se incrementa un 138, un 136 por 100 en Madrid y un 133 por 100 en Baleares. El número de años-personas vividos en Murcia y Extremadura disminuye, sin embargo, el riesgo de ruptura de la unión en relación a los vividos en Andalucía: en Murcia es un 45 por 100 menor y en Extremadura un 60 por 100.

Finalmente, cabe comentar que, en aras de dar respuesta al interrogante de si los determinantes sociodemográficos de las rupturas analizados actuaban de forma diferencial según se tratase de promociones de uniones antiguas o recientes, se testó el mismo modelo de regresión para las mujeres de tres grupos de promociones de uniones: 1941-60, 1961-65 y 1976-90. La agrupación de las promociones se basó en la selección en un mismo grupo de aquellas promociones que presentaban una mayor similitud en las curvas de sobrevivencia a la ruptura por duración de la unión. Los resultados de los tres modelos no indicaban diferencias significativas, los determinantes sociodemográficos de las rupturas actuaban en la misma dirección y con similar magnitud. Quizás es todavía demasiado pronto para encontrar diferencias significativas en el comportamiento de las generaciones en relación a un fenómeno cuya magnitud y evolución indican que se encuentra en las puertas de una más larga historia.

\section{A MODO DE CONCLUSIÓN}

El análisis descriptivo de la evolución de las rupturas en España y el análisis biográfico de los determinantes sociodemográficos aquí presentados confirmaron la mayoría de las hipótesis que se plantearon en los inicios de la investigación. El divorcio afecta selectivamente a una determinada parte de la población femenina: la más instruida y mejor situada en el mercado de trabajo. Si bien el impacto de la aprobación de la ley de divorcio es notable en la evolución de las rupturas, el cambio de evolución al alza fue anterior a la aprobación de esta ley y son observables, por otro lado, importantes efectos generacionales en su evolución.

En cuanto a los determinantes sociodemográficos del divorcio, los resultados encontrados para el caso español son consistentes con otras evidencias empíricas de otros países del Oeste europeo y de Estados Unidos en relación, 
sobre todo, a las variables que hacen referencia a las características de la unión. Pero en España parece ser un fenómeno socialmente más selectivo y, por ello, las variables educativas y laborales tienen un rol muy importante. Los resultados muestran que las rupturas de las uniones de las mujeres guardan una relación positiva con la existencia de un precedente de ruptura de unión paterno, con una categoría social de origen familiar elevada, con una promoción de la unión reciente, con una edad precoz de entrada en unión, con la cohabitación, con uniones no tradicionales en relación a las diferencias de edad entre los cónyuges, con la presencia de hijos, con un nivel de instrucción elevado, con una fuerte vinculación al mercado de trabajo, con la residencia en municipios de gran tamaño y con la residencia en determinadas Comunidades Autónomas.

Las hipótesis en relación al efecto de los hijos y a las diferencias que podrían esperarse en cuanto a los determinantes sociodemográficos según se tratase de uniones antiguas o recientes fueron refutadas. Por un lado, no era esperable que el riesgo de ruptura entre los que no tenían hijos fuera menor que entre los que tenían un hijo, controladas el resto de las variables sociodemográficas. Por otro lado, no parece encontrarse ninguna coherencia en los resultados de los diferentes modelos de análisis de regresión testados para ver la importancia no sólo del número de hijos, sino también de la edad de los mismos, en la propensión a una ruptura de la unión. Sin embargo, dada esta incoherencia de resultados, parece conveniente tomarlos con cautela y esperar a su confirmación en posteriores análisis. Tampoco se han encontrado diferencias significativas entre los determinantes sociodemográficos de las rupturas de las promociones antiguas y las más recientes Por último, se terminará señalando que se hace necesario acometer un estudio profundo de las posibles interacciones entre variables.

\section{BIBLIOGRAFÍA}

ANDERSON, G. (1995): «Divorce-risk trends in Sweden 1971-1993», European Journal of Population, 11, pp. 293-311.

Bumpass, L. L.; Castro Martín, T., y Sweet, J. A. (1991): "The Impact of Family Background and Early Marital Factors on Marital Disruption", Journal of Family Issues, vol. 12, núm. 1, pp. 22-42.

Castro Martín, T., y Bumpass, L. L. (1989): «Recent Trends in Marital Disruption», Demography, vol. 26, núm. 1, pp. 37-51.

DeLPHY, C. (1982): «Matrimonio y divorcio: el doble atolladero», Cuadernos Inacabados, 2-3, Barcelona, Edicions La Sal, pp. 65-76.

COMUnidAd DE MADRID/DepaRTAMENTO DE EsTAdísTiCa (1990): Informe sobre la situación de la estadística de divorcios, separaciones y anulaciones, Madrid, Consejería de Economía.

CONSEIL DE L'EuRope (1997): Évolution démographique récente en Europe. 1997, Strasbourg, Éditions du Conseil de l'Europe.

Dharmalingam, A.; Pool, I.; Hillcoat-Nalletamby, S., y McCluskey, N. (1998): Divorce in New Zealand, Paper presented at the 1998 PAA Conference, Chicago. 
Hoem, B., y Hoem, J. M. (1992): The Disruption of Marital and non-marital Unions in Contemporary Sweeden, en J. Trussel, R. Hankibnson y J. Tilton (eds.), Demographic Applications of Event History Analysis, Oxford, Clarendon Press, pp. 61-93.

Houle, R.; Simó, C.; Solsona, M., y Treviño, R. (1997): Informe analitic de la població separada y divorciada a Catalunya, pp. 1-32 (informe realizado para el Institut Català de la Dona en razón de un convenio de colaboración, mimeo).

Journal OF MARRIAGE AND THE FAMILY (1990): "Determinants of Divorce: A review of Research in the Eighties», Journal of Marriage and the Family, núm. 52, pp. 904-912.

Kellerhals et al. (1985): «Statut social, projet familial et divorce», Population, vol. 6.

Lesthaeghe, R. (1995): «The Second Demographic Transition in Western Countries. An Interpretation", en K. Oppenheim Mason y A. M. Jensen (eds.), Gender and Family Change in Industrialized Countries, Oxford, Clarendon Press.

Monnier, A., y GuiberT-Lantoine, C. de (1993): «La conjoncture démographique: l'Europe et les pays développés d'Outre-Mer», Population, 4, pp. 1043-1067.

Oppenheimer, V. K. (1994): "Women's Rising Employment and the Future of the Family in Industrial Societies», Population and Development Review, vol. 20, núm. 2, pp. 294-338.

Pinnelli, A., y Rose, A. de (1997): Micro and macro determinants of family formation and dissolution, paper presentado al XXIIIrd IUSSP General Population Conference, Beijing.

Roussel, L. (1993): «Sociographie du divorce et divorcialité», Population, 4, pp. 919-938.

RugGLeS, S. (1997): "The rise of divorce and separation in the United States, 1880-1990", Demography, vol. 34, núm. 4, noviembre, pp. 455-466.

Solsona, M. (1996): «La Segunda Transición Demográfica desde la perspectiva de género», en M. Solsona (ed.), Desigualdades de género en los viejos y los nuevos hogares. Aportaciones al Seminario "Gender Inequality in Old and New Households», Instituto de la Mujer, Ministerio de Trabajo y Asuntos Sociales, Estudios fuera de Colección, Madrid, pp. 17-46.

TzEnG, M. (1992): "The effects of socioeconomic heterofamy and changes on marital dissolution for first marriages", Journal of Marriage and the Family, vol. 54 (3), pp. 6049-19.

White, L. K. (1990): "Determinants of divorce: A Review of Research in the Eighties», Journal of Marriage and the Family, vol. 52, pp. 904-912.

Solsona, M.; SimÓ, C., y Houle, R. (1997): «Séparation et divorce en Espagne», XXIIIrd General Population Conference, IUSSP, Beijing, China (está publicado en Papers de Demografia, núm. 129, Centre d'Estudis Demogràfics).

Solsona, M.; René, H., y Simó, C. (1997): «Separation an Divorce in Spain», pp. 1-31 (de próxima publicación en la revista South European Society \& Politics).

\begin{abstract}
This paper presents the principle results of a current research project focusing on the sociodemographic determinants of divorce in Spain on the basis of the 1991 Sociodemographic Survey. It attempts to explain the propensity for the breakdown of first unions, which is shaped as a dependent variable, on the basis of a set of covariables which refer to the characteristics of the source family, individual characteristics and union characteristics. The authors then measure the effect of these co-variables on the probability of first-union disruption with a view to proposing a first socio-demographic picture of separation and divorce in Spain. A logistic regression discrete-time hazard model was used for the analysis.
\end{abstract}

